\title{
A hyperbolic shear and normal deformation theory for deflection and stresses of FGM sandwich plate
}

\author{
H. Saidi, A. Tounsi, A. E.A. Adda.Bedia \\ Laboratoire des Matériaux et Hydrologie, Université de Sidi Bel Abbes, Département de Génie Civil, Faculté de \\ Technologie, Algérie
}

\begin{abstract}
In the present paper, the static analysis of functionally graded sandwich plates subjected to thermo mechanical loads is studied. In this model, the displacements vary as a hyperbolic function through the thickness of the plate and satisfy stress boundary conditions on the top and the bottom of the plate. The material properties of the sandwich plate faces are assumed to be graded in the thickness direction according to a simple power-law distribution in terms of volume fraction of material constituents. The core layer is still homogeneous and made of an isotropic material. The governing equations of equilibrium for FG sandwich plates can be obtained using the virtual work principle, and the closed form solutions are obtained by using Navier technique. The accuracy of the present analysis is ascertained by comparing it with various results available in the literature. The influences played by side-to-thickness ratio, aspect ratio and volume fraction distributions are investigated.
\end{abstract}

\section{Introduction}

Functionally graded structures are those in which the volume fractions of two or more materials are varied continuously as a function of position along certain dimension(s) of the structure to achieve a required function. For example, thermal barrier plate structures for high-temperature applications may form from a mixture of ceramic and a metal. The composition is varied from a ceramic-rich surface to a metal-rich surface, with a desired variation of the volume fractions of the two materials in between the two surfaces. The ceramic constituent of the material provides the high-temperature resistance due to its low thermal conductivity [1]. The gradual change of material properties can be tailored to different applications and working environments. This makes functionally gradient materials preferable in many applications. The continuous change in the microstructure of Functionally Graded Materials (FGMs) distinguish them from the fibrereinforced laminated composite materials, which have a mismatch of mechanical properties across an interface due to two discrete materials bonded together.

FGMs are widely used in many structural applications such as mechanics, civil engineering, optical, electronic, chemical, mechanical, biomedical, energy sources, nuclear, automotive fields and ship building industries to eliminate stress concentration and relax residual stresses and enhance bond strength. These materials also have great potential in applications where the operating conditions are severe, including spacecraft heat shields, heat exchanger tubes, fly wheels, plasma facings for fusion reactors, biomedical implants, engine components, and high power electrical contacts or even magnets.

In the past few decades, various shear deformation theories have been proposed and implemented to analysis of plates. Moreover, increased use of advanced materials in primary structures necessitates the development of precise theoretical model to accurately predict the behavior of the structures.

The researchers have paid much attention for modeling of the plates over the past few decades and a variety of plate theories have been introduced. The CPT can only provide reasonable results for thin plates since it disregards the effects of the transverse shear deformation and transverse normal stress. The FSDT $[2,3]$ considers the effect of transverse shear deformation, but this theory needs a shear correction factor in order to satisfy zero transverse shear stress boundary conditions on the top and bottom. The various higher order shear deformation theories (HSDTs) were proposed to analyze the plates by Ambartsumian [4], Levinson [5], Touratier [6], Soldatos [7], Karama et al. [8], 
Aydogdu [9], Reddy [10], Xiang et al. [11] and Mantari et al. [12].

In the present paper, analysis of functionally graded sandwich plates under Thermo-mechanical loading is developed using a new hyperbolic shear strain shape function considering thickness stretching. This theory accounts for adequate distribution of the transverse shear strains in the thickness of the plate and satisfies the traction free boundary conditions on the top and bottom surface of the plates, thus a shear correction factor is not required. The accuracy of the present results is established by comparing those with well known trigonometric shear deformation theory.

\section{Geometry and material properties of functionally graded sandwich plate}

Let us consider the case of a sandwich plate composed of three microscopically heterogeneous layers as shown in Fig. 1. Rectangular Cartesian coordinates are used to describe infinitesimal deformations of a three-layer sandwich elastic plate occupying the region $[0, \mathrm{a}] \cdot[0, \mathrm{~b}] \cdot[\mathrm{h} / 2,+\mathrm{h} / 2]$ in the unstressed reference configuration. The midplane of the composite sandwich plate is defined by $\mathrm{z}=0$ and its external bounding planes being defined by $\mathrm{z}= \pm \mathrm{h} / 2$, the equations will be derived in tensorial notations and specialized afterwards for the problem under consideration. The face layers of the sandwich plate are made of an isotropic material with material properties varying smoothly in the $\mathrm{z}$ (thickness) direction only. The core layer is made of an isotropic homogeneous material.

The vertical positions of the bottom and top, and of the two interfaces between the layers are denoted by $h 0=-h / 2, h 1, h 2, h 3=h / 2$. The effective material properties for each layer, like Young's modulus and Poisson s ratio, can be expressed as:

$$
P^{(n)}(z)=P_{m}+\left(P_{c}-P_{m}\right) V^{(n)}
$$

where $\mathrm{P}_{\mathrm{m}}$ and $\mathrm{Pc}$ denote the property of the bottom and top faces of layer 1, respectively, and vice versa for layer 3 depending on the volume fraction $V^{(n)}(\mathrm{n}$ $=1,2,3)$. Note that Pm and Pc are, respectively, the corresponding properties of the metal and ceramic of the FGM sandwich plate. The volume fraction $V^{(n)}$.

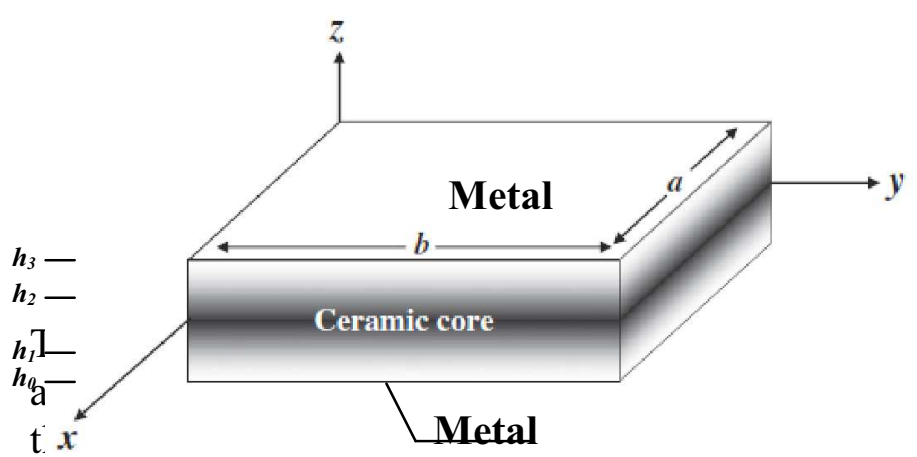

Figure1. Geometry of the FGM sandwich

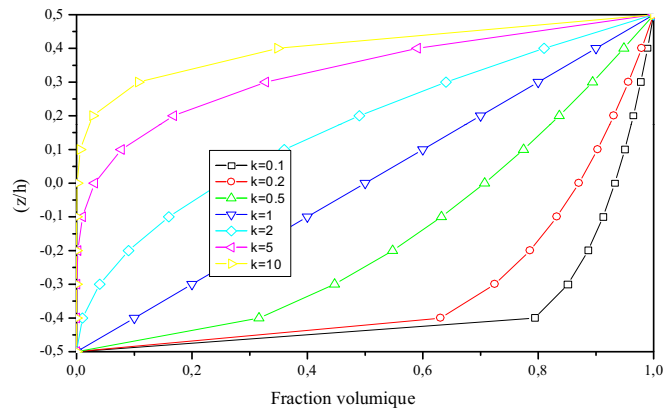

Figure 2: Variation in the volume fraction in a plate P-FGM.

\section{Mathematical formulation and analytical solution}

The displacement field for FG sandwich plates is described in the following equations:

$u(x, y, z)=u_{0}(x, y)-z w_{, x}+f(z) \theta_{x}$
$v(x, y, z)=v_{0}(x, y)-z w_{, y}+f(z) \theta_{y}$
$w(x, y, z)=w_{0}(x, y)+\grave{f}(z) \theta_{z}$

where, $\boldsymbol{u}, \boldsymbol{v}, \boldsymbol{w}$ are displacements in the $\boldsymbol{x}, \boldsymbol{y}, \boldsymbol{z}$ directions, $\boldsymbol{u}_{0}, \quad \boldsymbol{v}_{0}$ and $\boldsymbol{w}_{0}$ are midplane displacements, $\boldsymbol{\theta}_{\boldsymbol{x}}, \boldsymbol{\theta}_{\boldsymbol{y}}$ and $\boldsymbol{\theta}_{\boldsymbol{z}}$ rotations of the $y z$, $x z$, and $x y$ planes due to bending, respectively. $f(z)$ represents shape function determining the distribution of the transverse shear strains and stresses along the thickness and $\boldsymbol{f}^{\prime}(\boldsymbol{z})=\partial \boldsymbol{f}(\boldsymbol{z}) / \partial \boldsymbol{z}$.

The linear strain expressions derived from the displacement model are as follows: 
$\left\{\begin{array}{l}\gamma_{y z} \\ \gamma_{x z}\end{array}\right\}=f^{\prime}(z)\left\{\begin{array}{l}\gamma_{y z}^{0} \\ \gamma_{x z}^{0}\end{array}\right\}$

The linear constitutive relations are given as:

$$
\left\{\begin{array}{c}
\sigma_{x} \\
\sigma_{y} \\
\sigma_{z} \\
\tau_{y z} \\
\tau_{x z} \\
\tau_{x y}
\end{array}\right\}=\left[\begin{array}{cccccc}
\boldsymbol{Q}_{11} & \boldsymbol{Q}_{12} & \boldsymbol{Q}_{13} & 0 & 0 & 0 \\
\boldsymbol{Q}_{12} & \boldsymbol{Q}_{22} & \boldsymbol{Q}_{23} & 0 & 0 & 0 \\
\boldsymbol{Q}_{13} & \boldsymbol{Q}_{23} & \boldsymbol{Q}_{33} & 0 & 0 & 0 \\
0 & 0 & 0 & \boldsymbol{Q}_{44} & 0 & 0 \\
0 & 0 & 0 & 0 & \boldsymbol{Q}_{55} & 0 \\
0 & 0 & 0 & 0 & 0 & \boldsymbol{Q}_{66}
\end{array}\right]\left\{\begin{array}{c}
\boldsymbol{\varepsilon}_{x} \\
\boldsymbol{\varepsilon}_{y} \\
\boldsymbol{\varepsilon}_{z} \\
\gamma_{y z} \\
\gamma_{x z} \\
\gamma_{x y}
\end{array}\right\}
$$

where $\left(\sigma_{x}, \sigma_{y}, \sigma_{z}, \tau_{y z}, \tau_{x z}, \tau_{x y}\right)$ and $\left(\varepsilon_{x}, \varepsilon_{y}, \varepsilon_{z}\right.$, $\left.\gamma_{y z}, \gamma_{x z}, \gamma_{x y}\right)$ are the stress and strain components, respectively, $Q_{i j}$ are the stiffness coefficients.

The principle of virtual work, the following expressions can be obtained:

$$
\int_{-h / 2 \Omega}^{h / 2}\left[\sigma_{x} \delta \varepsilon_{x}+\sigma_{y} \delta \varepsilon_{y}+\sigma_{z} \delta \varepsilon_{z}+\tau_{x y} \delta \gamma_{x y}+\tau_{y z} \delta \gamma_{y z}+\tau_{x z} \delta \gamma_{x z}\right] d \Omega d z-\int_{\Omega} q \delta
$$

The governing equations of equilibrium can be derived easily from Equation (5)

$$
[C]\{A\}=\{F\}
$$

where

$\{\Delta\}=\{U, V, W, X, Y, Z\}^{t}$ and $[C]$ is the stiffness matrix, $\{F\}$ is the force vector.

\section{Numerical example and discussion}

Numerous examples are solved to ensure the accuracy of the present theory for the prediction of static analysis under thermo mechanical loading, the closed form solution are obtained using the Navier solution of simply supported FGM sandwich plates.

The characteristic values for metal and ceramics used in the FG sandwich plate are listed in Table 1.
Table1. Material properties used in the FGM plate.

\begin{tabular}{|l|l|l|}
\hline Properties & Metal (Aluminium) & Ceramic \\
\hline $\mathrm{E}(\mathrm{GPa})$ & 70 & 380 \\
\hline$v$ & 0.3 & 0.3 \\
\hline$\rho\left(\mathrm{Kg} / \mathrm{m}^{3}\right)$ & 2707 & 3800 \\
\hline
\end{tabular}

Figures 3 and 4 show the variation of the dimensionless deflection $\bar{w}$ with side-tothickness ratio $a / h$ and the aspect ratio $a / b$ for one type of sandwich symmetric square plates (hFGM=1).

The deflection is maximum for the metallic plate and minimum for the ceramic plate. It decreases with the increase of $a / h$ and $a / b$ ratios.

Figures 5 and 6 show plots of the through-thethickness distribution of the transverse shear stress $\bar{\tau}_{x z}$ and axial stress for $\boldsymbol{k}=0.0,1.5$, and 3.5 of symmetric sandwich square plates.

The maximum value occurs at a point on the mid-plane of the plate and its magnitude for FGM sandwich plate is larger than that for homogeneous ceramic plate.

In Figures 7 and 8, we have plotted the throughthe-thickness distributions of the dimensionless axial stress $\bar{\sigma}_{x}$ and the deflection $\bar{w}$ of the FG sandwich plate for $\boldsymbol{k}=3.5$ and $h F G M=1$, respectively. These figures show the great influence played by the different thermal and bending loads on the axial stresses and deflection.

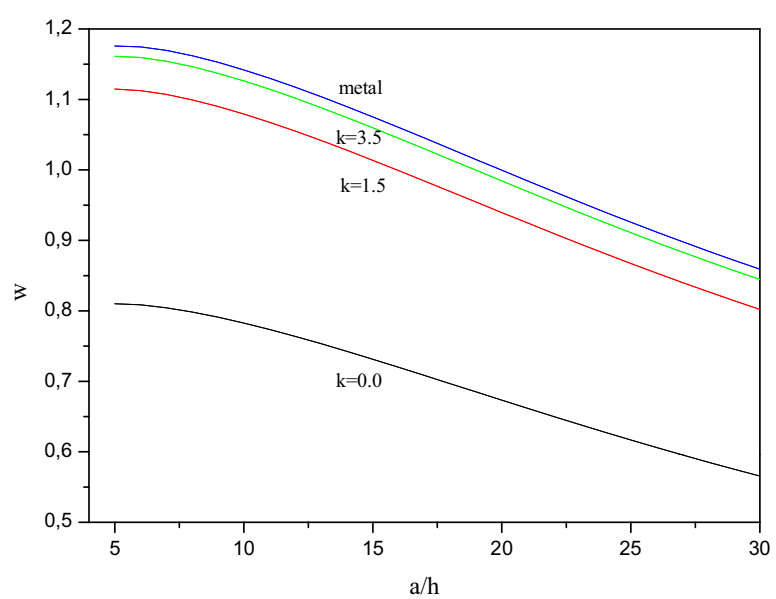

Figure3 Dimensionless center deflection w vs a function of side to thickness ratio $\mathrm{a} / \mathrm{h}$ for FGM sandwich plate $(\mathrm{hFGM} / \mathrm{h}=1)$ 


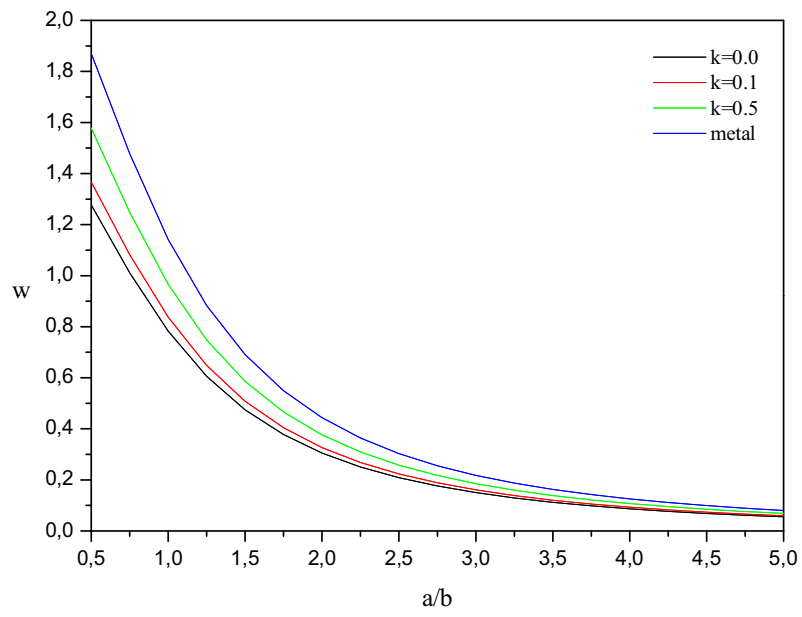

Figure 4 Effect of the aspect ratio $a / b$ on the dimensionless the center deflection $w$ of FGM sandwich plates $(\mathrm{hFGM}=1$ )

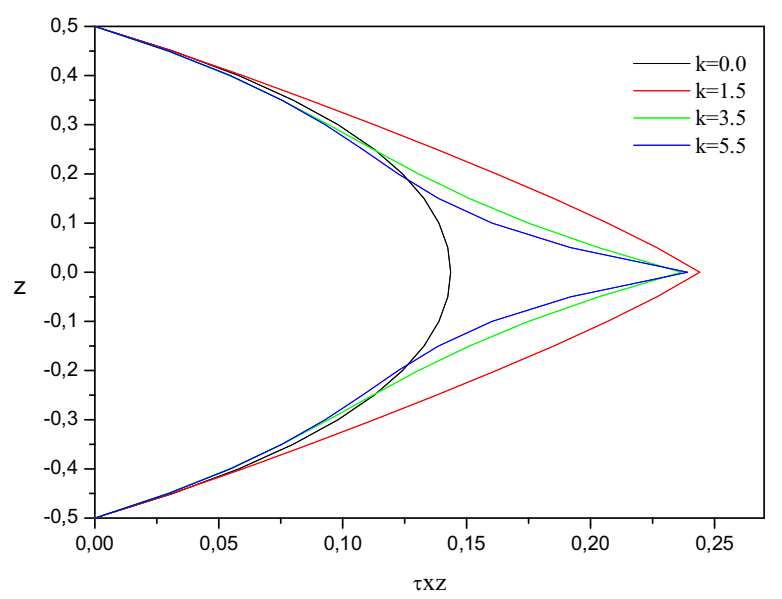

Figure 5 Variation of transverse shear stress $\tau \mathrm{xz}$ through the plate thickness for FGM sandwich plate $(\mathrm{hFGM}=1)$

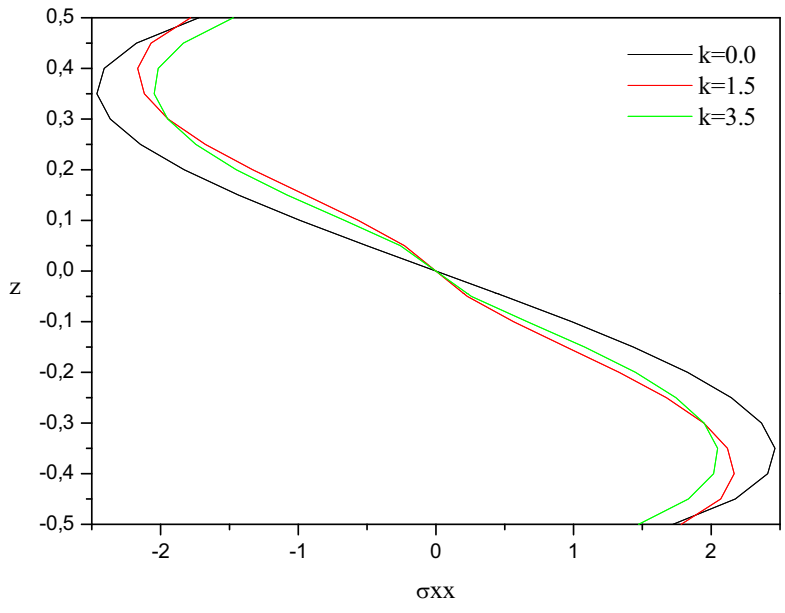

Figure6 Variation of axial stress sigmax through theplate thickness for FGM sandwich plate (hFGM=1)

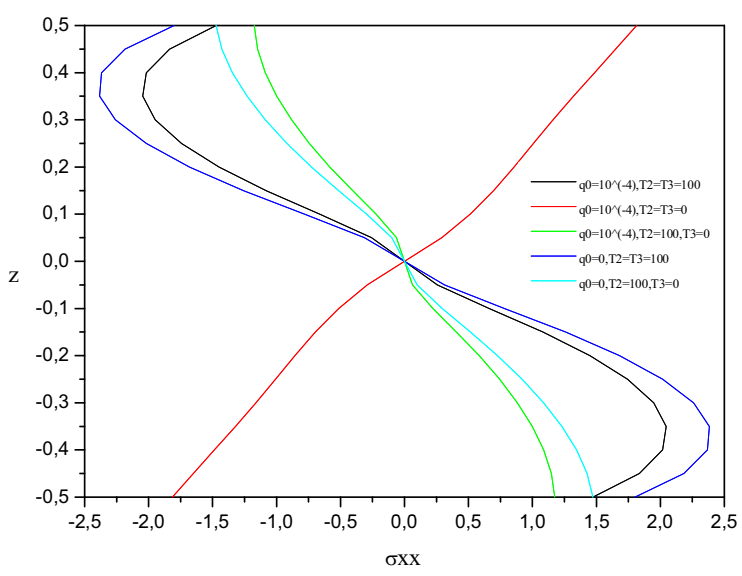

Figure 7 Effect of the thermal load $\mathrm{T}_{3}$ on axial stress $\sigma \mathrm{xx}$ of the FGM sandwich plate (hFGM=1, k=3.5)

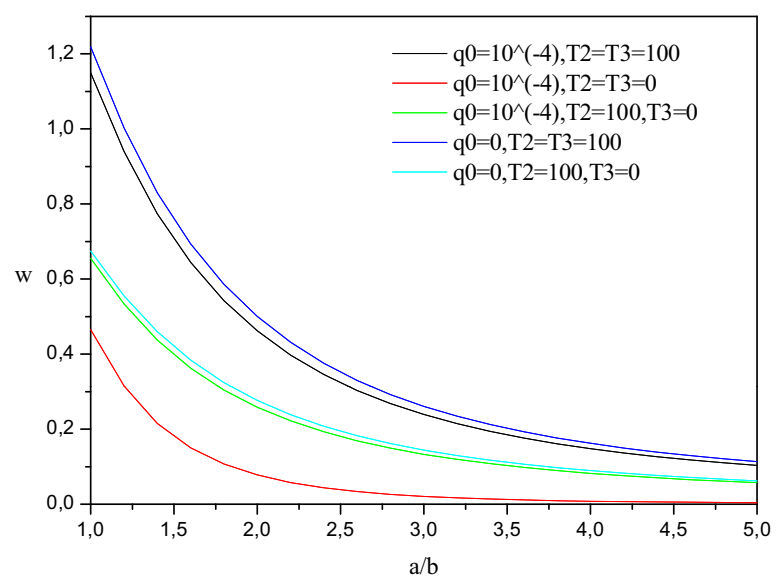

Figure8 Effect of mechanical and temperature loads on the dimensionless center deflection of an FGM sandwich plate $(\mathrm{hFGM}=1, \mathrm{k}=3.5)$ 


\section{Conclusion}

The static analysis of functionally graded sandwich plates subjected to thermo mechanical loads is studied. The displacement and stresses have been analyzed under thermo mechanical loadings. The gradation of the properties through the thickness is assumed to be of the power law distribution in terms of volume fractions of the constituents, the core layer is made of ceramic.

The governing equations are derived by employing the principle of virtual work. These equations are then solved via Navier -type solution. The results are presented for one type of simply supported rectangular sandwich plates under thermo-mechanical loading. The accuracy of the present analysis is ascertained by comparing it with various results available in the literature. The influences played by side-tothickness ratio, aspect ratio and volume fraction distributions are investigated.

\section{REFERENCES}

[1] Hasselman DPH, Youngblood GE. Enhanced thermal stress resistance of structural ceramics with thermal conductivity gradient. Journal of the American Ceramic Society 1978; 61(1,2):49\{53.

[2] Reissner E. Reflection on the theory of elastic plates. J Appl Mech 1945;38:1453-64.

[3] Mindlin RD. Influence of rotary inertia and shear on flexural motions of isotropic elastic plates. J Appl Mech 1951;18:31-8.

[4] Ambartsumian SA. On the theory of bending plates. Izv Otd Tech Nauk AN SSSR 1958;5:69-77.

[5] Levinson M. An accurate simple theory of static and dynamics of elastic plates. Mech Res Commun 1980;7:343-50.

[6] Touratier M. An efficient standard plate theory. Int J Eng Sci 1991;29:901-16.

[7] Soldatos KP. A transverse shear deformation theory for homogeneous monoclinic plates. Acta Mech 1992;94:195200.

[8] Karama M, Afaq S, Mistou S. Mechanical behavior of laminated composite beam by new multi-layered laminated composite structures model with transverse shear stress continuity. Int J Solids Struct 2003;40:1525-46.
[9] Aydogdu M. A new shear deformation theory for laminated composite plates. Compos Struct 2003;89:94-101.

[10] Reddy JN. Mechanics of laminated composite plates and shells: theory and analysis. 2nd ed. Boca Raton: CRC Press; 2004. [11] Xiang S, Jin YX, Bi ZY, Jiang SX, Yang MS. A n-order shear deformation theory for free vibration of functionally graded and composite sandwich plates.

Compos Struct 2011;93:2826-32.

[12] Mantari JL, Oktem AS, Soares CG. A new higher order shear deformation theory for sandwich and composite laminated plates. Composites Part B, 2012;43:1489-99. 